\title{
Quasi-Particle Degrees of Freedom versus the Perfect Fluid as Descriptors of the Quark-Gluon Plasma
}

\author{
L.A. Linden Levy, J.L. Nagle, and C. Rosen \\ University of Colorado, Boulder \\ P. Steinberg \\ Brookhaven National Laboratory
}

(Dated: October 26, 2018)

\begin{abstract}
The hot nuclear matter created at the Relativistic Heavy Ion Collider (RHIC) has been characterized by near-perfect fluid behavior. We demonstrate that this stands in contradiction to the identification of QCD quasi-particles with the thermodynamic degrees of freedom in the early (fluid) stage of heavy ion collisions. The empirical observation of constituent quark " $n_{q}$ " scaling of elliptic flow [1] is juxtaposed with the lack of such scaling behavior in hydrodynamic fluid calculations followed by Cooper-Frye freeze-out to hadrons. A "quasi-particle transport" time stage after viscous effects break down the hydrodynamic fluid stage, but prior to hadronization, is proposed to reconcile these apparent contradictions. However, without a detailed understanding of the transitions between these stages, the " $n_{q}$ " scaling is not a necessary consequence of this prescription. Also, if the duration of this stage is too short, it may not support well defined quasi-particles. By comparing and contrasting the coalescence of quarks into hadrons with the similar process of producing light nuclei from nucleons, it is shown that the observation of " $n_{q}$ " scaling in the final state does not necessarily imply that the constituent degrees of freedom were the relevant ones in the initial state.
\end{abstract}

PACS numbers: 25.75.Dw

\section{INTRODUCTION}

The understanding that hadrons are not fundamental particles, but rather composite systems comprised of asymptotically-free quarks and gluons, led directly to the idea that hadronic matter under extreme conditions of temperature and pressure would transform into a "quarkgluon plasma" [2]. In this state, quarks and gluons could be thought of as quasi-free particles moving along trajectories, such that the thermodynamic properties could be approximated as that of an ideal gas with non-interacting degrees of freedom. More specifically, quarks and gluons would no longer be confined in their parent hadron, but would be free to roam about larger volumes. This scenario gives 40 degrees of freedom for the quark and gluon states (including two light quarks [53]) as opposed to 3 pion degrees of freedom in a hadronic system at a lower temperature. This enumeration is often associated with the order of magnitude change in the entropy $s / T^{3}$ and energy density $\epsilon / T^{4}$ as observed near the transition temperature in lattice QCD calculations [3] .

This physical picture has generally been thought to be naive, since the high densities and moderate momentum transfers should lead to short mean free paths. However, it has a certain persistence in the heavy ion literature (e.g. the CERN press release [4] or the discussion of such in the review [5]) as it presents an easily-described physical scenario with nominally well-defined consequences. Over the last few years, experimental data has suggested a paradigm shift where the quark-gluon plasma is described as a nearly inviscid fluid, and yet the picture of quasi-particles carrying the thermodynamic degrees of freedom often persists. Notably, recent experimental papers describing the scaling properties of elliptic flow measurements state that "scaling suggests that partonic collectivity dominates the transverse expansion dynamics [6]" and "[scaling indicates] a pre-hadronization state in which the flowing medium reflects quark degrees of freedom [7]." The analysis presented in this paper suggest that these conclusions are not well supported either by experimental data or theoretical ideas.

\section{QUASI-PARTICLES IN THE QUARK-GLUON PLASMA}

Interactions between propagating quarks and gluons in the quark-gluon plasma generates thermal masses, and thus the quarks and gluons can be called "quasiparticles." A quasi-particle's mass is determined by the local properties of the medium, which thus distinguishes between Lorentz frames. By contrast a "real" particle has a Lorentz-invariant rest mass. Such quasiparticle descriptions allow even strongly coupled systems to show properties reminiscent of free non-interacting systems. The characteristic feature which indicates socalled "good" quasi-particle states is a decay width (which characterizes the coupling strength and number of interaction channels) that is smaller than its mass.

The dynamical role of quasi-particles in the hot phase of QCD is under intense debate. In [8] an accurate match to lattice QCD calculations of the entropy for a purely gluonic medium is obtained in a perturbative calculation involving quasi-particles for temperatures down to $2 T_{c}$. 
They note that "although the quasiparticle picture suggested by such fits is a rather crude representation of the actual physics of non-abelian gauge theories, it supports the idea that one should be able to give accurate description of thermodynamics of the QCD plasma in terms of its elementary excitations." Such quasi-particle models have been extended and applied down to $1.05 T_{c}$ where they are able to describe deviations from the free quark and gluon picture 9, 10]. However, they find that these effective degrees of freedom are rather heavy and have a sizable width making it difficult to associate them with "strict" quasi-particles. Thus, it is not obvious that the identification of the hot dense system with a quark-gluon plasma necessarily implies a quasi-particle picture, even where perturbative calculations are possible.

Additional information on quasi-particles near the transition temperature are available from lattice QCD. The suggestion that the bulk thermodynamics of the quark-gluon plasma are governed by a large set of colored bound states of light quarks [11 has largely been ruled out by examining fluctuations of quark number and electric charge [12]. Additionally, baryon-strangeness correlations place severe limits on $q \bar{q}$ bound states $[13,14]$. It is notable that the consistency of $\chi_{u d}$ with a weakly interacting plasma of quarks and anti-quarks above $T_{c}$ 14] does not provide compelling evidence that these are well defined quasi-particles dominating the bulk thermodynamics.

In many systems the thermodynamic degrees of freedom can be counted by associating them with particles or quasi-particles and accounting for their thermal motion and quantum numbers (for example $\frac{3}{2} k T$ and $\frac{5}{2} k T$ for non relativistic monatomic gases and diatomic gases respectively). A key question is whether or not this is the case for the quark-gluon plasma. The thermodynamic properties of some systems, such as Landau's Fermi liquid [15], are dominated by the quasi-particle degrees of freedom, while in other systems quasi-particles are a single medium excitation (for example the fractional quantum hall effect [16]). In the case of hot QCD matter both may be interesting, but the former is the more relevant when discussing the quark-hadron phase transition observed on the lattice.

\section{FREEZE-OUT FROM HYDRODYNAMICS}

One of the great discoveries of the RHIC program has been that simple hydrodynamic models provide a good description of RHIC data, especially the development of radial and elliptic flow, see [17] and references therein. The hydrodynamic equations are based on the energymomentum tensor, and viscosity is included as an expansion parameter in terms of velocity gradients. The viscosity term is set to zero for a mathematically perfect fluid. When invoking a picture of quasi-particles, a connection can be made between the mean free path of these objects via kinetic theory and the viscosity term in the hydrodynamic equations. The hydrodynamic limit of the quasi-particle picture is reached when the typical gradients in the flow involve length scales that are large compared to the typical mean free paths.

First-order estimates suggest that experimental data is consistent with essentially zero viscosity [18], but cannot rule out a viscosity to entropy density ratio consistent with the lower bound derived from the AdS/CFT duality $(\eta / s \geq 1 / 4 \pi)[19]$. Furthermore, estimates of collective behavior based on classical transport (Boltzmann) approaches are generally unable to describe RHIC data, whether due to the small cross sections implied by a perturbative transport picture [20], or the large formation times required by hadronic transport [21].

The hydrodynamic behavior of the medium must eventually break down as the density of the medium drops below a threshold value, and particles "freeze out" into vacuum. In addition, there may be particles (for example at high transverse momentum) that are not equilibrated into the bulk medium during the limited evolution time $(t<10-15 \mathrm{fm} / \mathrm{c})$ [22]. Thus, in order to compare hydrodynamic calculations with experimental data (i.e. measured hadron distributions after freezeout), hydrodynamic calculations are typically terminated at a chosen temperature $T$. This is usually chosen to be the Hagedorn temperature $T_{c h} \sim 170 \mathrm{MeV}$ in single freeze-out models, and $T_{t h} \sim 100 \mathrm{MeV}$ when a thermal freeze-out temperature is postulated due to hadronic re-scattering. In either case, this prescription defines a "freeze-out hyper-surface" in space-time, also referred to as a "surface of last scattering." The Cooper-Frye formalism [23] is then used to decay fluid elements to hadrons by assuming local statistical hadronization. Many calculations also include the important non-equilibrium rescattering between hadrons after hadronization up to the point of freeze-out 24, 25].

Shown in Figure 1 are the published experimental data [1, 26, 27] on elliptic flow $v_{2}$ as a function of transverse momentum for various hadron species in minimum bias $A u+A u$ collisions at $\sqrt{s_{N N}}=200 \mathrm{GeV}$. Also shown are the results of a particular hydrodynamic calculation terminated with Cooper-Frye freeze-out 28]. This calculation begins with an equation of state that assumes chemical equilibrium until kinetic freeze-out (EoS Q), and a first order phase transition at $T_{c}=165 \mathrm{MeV}$. The initial time is taken as $\tau=0.6 \mathrm{fm} / c$, and freeze-out occurs when the temperature drops to $T_{f_{o}}=130 \mathrm{MeV}$. The particle spectra and elliptic flow $\left(v_{2}\right)$ qualitatively agree with the experimental data for values of $p_{T}<1.5 \mathrm{GeV} / c$.

The reasonable agreement of these calculations with the data, even without an extensive parameter sensitivity or error analysis, has generally been taken as evidence that collisions of nuclei at RHIC form a perfect inviscid fluid (i.e. small $\eta / s$ ) with short thermalization times 


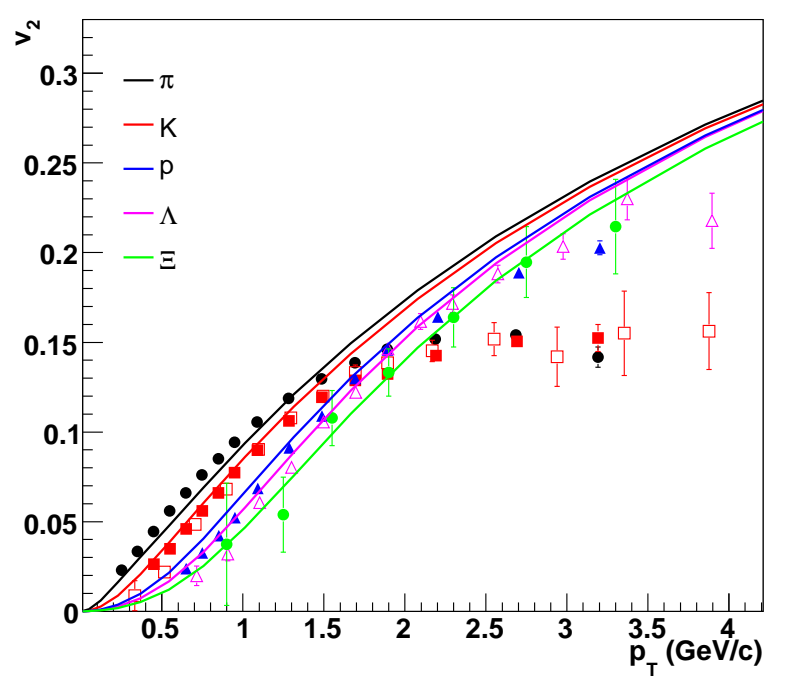

FIG. 1: (color online) Elliptic flow $v_{2}$ for identified hadrons as a function of $p_{T}$ in minimum bias $A u+A u$ collisions at $\sqrt{s_{N N}}=200 \mathrm{GeV}$, compared with hydrodynamic calculations assuming Cooper-Frye freeze-out.

$\left(\tau_{0}<0.6 \mathrm{fm} / \mathrm{c}\right)$. Notably, different calculations with different equations of state or initial conditions do not all agree with the data at the same level [29]. Similarly small values of $\eta / s$ have also been derived via measurements of charm suppression and flow [30]. Of course, the quantitative importance of viscosity has only started to get serious treatment, both experimentally and theoretically [18, 31, 32].

It is common in the field for the full range of transverse momenta to be sub-divided into three categories: The "low $p_{T}$ " region $\left(p_{T}<1.5 \mathrm{GeV}\right)$ where hydrodynamics agrees with experimental data; the "intermediate $p_{T}$ " region $\left(p_{T} \approx 1.5-4.5 \mathrm{GeV} / c\right)$ where hydrodynamics over-predicts elliptic flow $v_{2}$; and the "high $p_{T}$ " region $\left(p_{T}>4.5 \mathrm{GeV}\right)$ where hadronization from jet fragmentation is thought to be dominant. In the "intermediate $p_{T}$ " region, the violation of "hydrodynamic scaling" and the observation of enhanced (anti) baryon relative to meson yields (often referred to as the "baryon anomaly" 29, 33, 34]) led some to postulate a different hadronization mechanism.

\section{RECOMBINATION AND " $n_{q}$ " SCALING}

It has recently been speculated that in RHIC collisions [35] a thermal distribution of quarks might coalesce (a process sometimes referred to as "recombination") into hadrons when they satisfy the minimum quantum number requirements. If these requirements are met in a local region of phase space, then only the valence quarks of the correct flavors (and no additional constraints) are

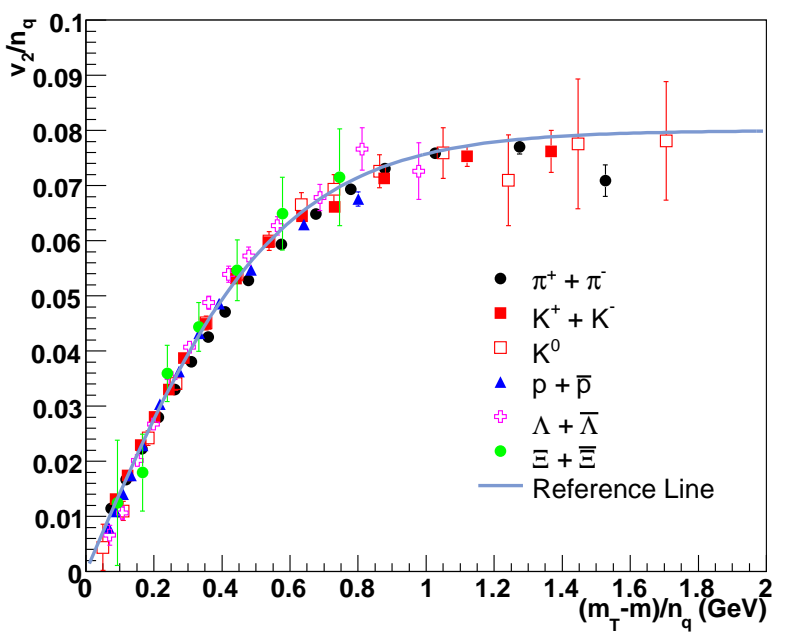

FIG. 2: (color online) The same data as in Figure 1, plotted as $v_{2}$ vs. $m_{T}-m$, the hadron transverse kinetic energy, both scaled by the number of constituent quarks per hadron. The reference line is a fit to the kaon data.

needed to form a hadron. In [33], they argue that in the light-cone gauge the hadron wave-function can be expanded in a Fock space of quarks and anti-quarks where the first order term is simply the valence quark content (e.g. $|p\rangle=|u u d\rangle+|u u d d \bar{d}\rangle+\ldots)$, and that this is applicable when $p_{T} \gg m$ for the hadron. This simple picture has been successful in qualitatively understanding the "baryon anomaly" and flow patterns. Interestingly, this "recombination" picture appears to involve a decrease in entropy; although, for "intermediate $p_{T}$ " hadrons one can always posit that compensating additional entropy is generated in the bulk medium at lower $p_{T}$. In fact, it has been suggested in [36] that the lattice QCD equation of state permits isentropic hadronization.

It has been empirically observed [1] that all hadrons follow a universal trend when $v_{2}$ versus $p_{T}$ is represented instead as $v_{2} / n_{q}$ versus $\left(m_{T}-m\right) / n_{q}$, where $n_{q}$ is the number of valence quarks in the hadron formed and $m_{T}=\sqrt{p_{T}^{2}+m^{2}}$ is the transverse mass. This was shown with STAR and PHENIX data [1] (as reproduced here) in Figure 2, and expressed as a function of the transverse kinetic energy $K E_{T}=m_{T}-m$. This scaling is often termed "constituent quark scaling."

If there are quasi-particles with the quantum numbers of quarks that are coalescing into hadrons, then an immediate question is what are the values for the thermal masses and widths. The "constituent quark" nomenclature implies an effective mass of $m_{N} / 3 \approx 300 \mathrm{MeV}$. However, the $300 \mathrm{MeV}$ constituent quark mass in a proton is generated by the spontaneous breaking of chiral symmetry $(\langle q \bar{q}\rangle \neq 0)$. Lattice QCD results indicate an approximate restoration of chiral symmetry in the quarkgluon plasma region above the critical temperature [37]. 


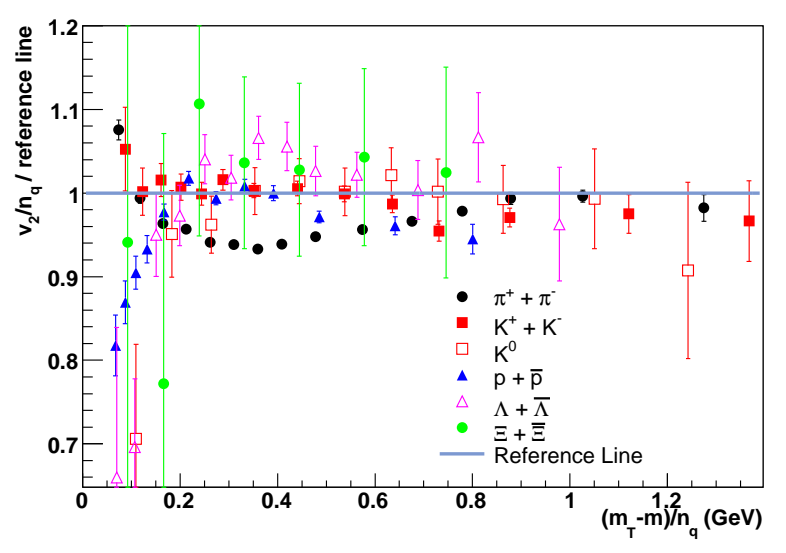

FIG. 3: (color online) The same data as in Figure 2, divided by a reference function fit as described in the text.

Any such quasi-particles would be expected to have an effective mass, but not from chiral symmetry breaking. Therefore the light quark masses assigned in [38] seem a remarkable coincidence since the dynamical mass of order $g T$ that might be generated in the thermal medium does not violate chiral symmetry and is quite different from a constituent quark mass. In addition, since the scaling appears to work for mesons (including the pions, which are anomalously light Goldstone bosons), a simple coalescence of a $300 \mathrm{MeV}$ quark and a $300 \mathrm{MeV}$ anti-quark easily violates local energy-momentum conservation.

If the quasi-particles containing different flavor quantum numbers (e.g. light quark flavor versus strange) have different masses, some scaling violations are expected [39]. However, in the limit that the mass of such quasi-particles is much less than their momentum, the scaling with $n_{q}$ gives no information about the mass differences. In the case of charm flavor quasi-particles, the current quark mass difference $(\sim 1.4 \mathrm{GeV})$ is always large compared with $g T$ and the momentum up to intermediate $p_{T}$, so future measurements of scaling agreement or violation are particularly interesting [39, 40].

Despite the categorization of the dominant physics in different $p_{T}$ ranges, the scaling does seem to work well at all $p_{T}$. To examine this quantitatively, the data is fit by a reference curve of the form $v_{2} / n_{q}=\alpha \tanh \left(\beta\left(m_{T}-\right.\right.$ $m) / n_{q}$ ) (as shown in Figure 21), where the parameters $\alpha$ and $\beta$ are determined from the charged kaon data alone. Fitting the kaon data is arbitrary and thus when dividing by the reference line, one only obtains a measure of the magnitude of deviation of the different hadrons from each other as shown in Figure 3 .

The scaling behavior for $\left(m_{T}-m\right) / n_{q}$ appears to hold at the $\pm 5 \%$ level for all hadron species measured for $\left(m_{T}-m\right) / n_{q}>0.2 \mathrm{GeV}$. Note that $\left(m_{T}-m\right) / n_{q}=0.2$ $\mathrm{GeV}$ corresponds to $p_{T}=0.52,0.75$, and $1.21 \mathrm{GeV} / c$ for pions, kaons and protons respectively. Below this value,

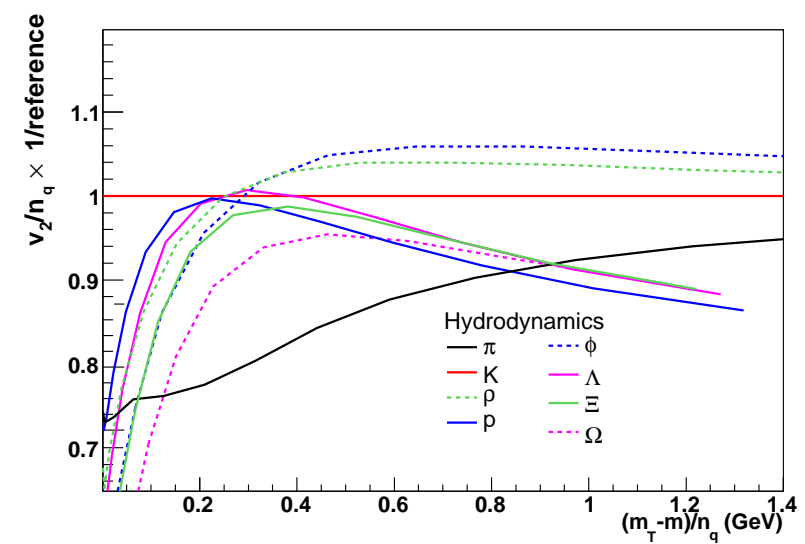

FIG. 4: (color online) The same hydrodynamic calculations shown in Fig. 1 , shown as $v_{2} / n_{q}$ vs. $\left(m_{T}-m\right) / n_{q}$. Note the absence of the scaling seen in the data.

the deviations are larger (e.g. $\pm \% 15-25 \%$ ); It is notable that the charged and neutral kaons have the largest deviation from each other, which may indicate some experimental issues. We note that there are first measurements of other hadrons (for example $\phi[\underline{6}, 7]$ and $\Omega$ and light nuclei $d$ and ${ }^{3} \mathrm{He}[6,41]$ ), but the current errors only allow one to confirm general agreement with the scaling behavior.

The " $n_{q}$ " scaling at low $p_{T}$ is not a natural prediction of hydrodynamics followed by Cooper-Frye freeze-out. These calculations depend only on the particle masses, and not on the quark content. However, it has been noted that if there is a regime where $v_{2}$ is linear as a function of $m_{T}-m$, then any re-scaling $v_{2} / \alpha$ and $\left(m_{T}-m\right) / \alpha$ will work for any constant $\alpha$ [42]. In order to test this, in Figure 4, a hydrodynamic calculation is plotted as a function of the scaling variables $v_{2} / n_{q}$ and $\left(m_{T}-m\right) / n_{q}$. Again, the different hadron species have been compared to the kaons, representing an arbitrary choice of reference. It is notable that the hydrodynamic calculation results are sensitive to the choice of initial conditions and equation of state. However, we do not expect these variations or the addition of hadronic re-scattering to modify the scaling results. The results reveal $\pm 15-25 \%$ level deviations of the various hadrons from the reference line; whereas, the experimental data shown on the identical scale in Figure 3 do not. The hydrodynamic calculations do not have a linear relation between these two variables even at low $p_{T}$, as a function of either $m_{T}-m$ or $p_{T}$, at a level that precise experimental data has tested.

As seen in the Figure 3, it appears that the data follow the $n_{q}$ scaling hypothesis within experimental uncertainties above $\left(m_{T}-m\right) / n_{q} \approx 0.2 \mathrm{GeV}$. The hydrodynamic calculation shown in Figure 4does not, particularly in the so-called "hydrodynamic regime." This observation does not necessarily imply that a full recombination calcula- 
tion would follow the " $n_{q}$ " scaling as well as the data, but it is clear that hydrodynamics fails to predict this emperical observation.

\section{RESONANCE CONTRIBUTIONS}

The level of agreement of the elliptic flow data with " $n_{q}$ " scaling is quite surprising, considering all of the reasons that exist for it not to work (for example see [43]). In fact, if all hadrons are formed via recombination of constituent quarks, then one might expect that $\rho$ mesons and $\Delta$ resonances would follow the scaling, but their decay daughter products might not [4]. The decay of these resonances results in pions with a distorted $v_{2}$ scaling, since the decay blurs the emission angle and shifts the $p_{T}$ of the pion relative to that of the parent resonance. We have quantified this effect using a Monte Carlo simulation. We show in Figure 5 ] the $v_{2} / n_{q}$ for $\pi$ from $\rho$ decay, $\pi$ from $\Delta$ decay, and pions from all sources combined. In the simulation, $\Delta, \rho$ and $\pi$ particles are given a $p_{T}$ according to the spectra calculated in [28] with hydrodynamics. In our simulation, the $\rho$ and $\Delta$ resonances, and the directly hadronized non-decay pions are assumed to follow the "universal" scaling curve (reference line) drawn in Figure 2. The $\rho$ and $\Delta$ resonances are subsequently allowed to decay, and the flow of the pions is computed. Shown in Figure 6 are the simulation results divided by the original reference line. Interestingly, the $\pi$ from $\Delta$ decay has a significantly larger $v_{2}$ than the scaling, as previously noted in [4]. In fact, by construction, the elliptic flow for $\pi$ from $\Delta$ decay must asymptotically approach 1.5 times the thermal pion value at large $p_{T}$ where the decay products are essentially co-linear with the parent. This large deviation in the " $n_{q}$ " scaling is deemphasized in the combined result $\left(\pi_{\text {all }}\right)$ due to the small fraction of $\Delta$ decay pions in the sample. In contrast, we find that $\pi$ from $\rho$ decay have a slightly reduced $v_{2}$ below $\left(m_{T}-m\right) / n_{q}<0.7 \mathrm{GeV}$. In fact, when the different contributions are combined, the scaling law appears "accidentally" obeyed at the $10 \%$ level with a deviation in a region of $\left(m_{T}-m\right) / n_{q}$ similar to that observed in the experimental data.

\section{QUASI-PARTICLE IMPLICATIONS}

Assuming that quasi-particles with the quantum numbers of quarks exist in the bulk presents some theoretical interpretation challenges since the light-cone gauge calculation assumes hadrons with $p_{T} \gg m$ and moves the entropy issue to lower $p_{T}$. In addition, it has been noted by many authors that the scaling is not an obvious consequence of hadronization via quark coalescence, but instead a consequence of the phase space distributions of the recombining quarks, e.g. as shown in [43].

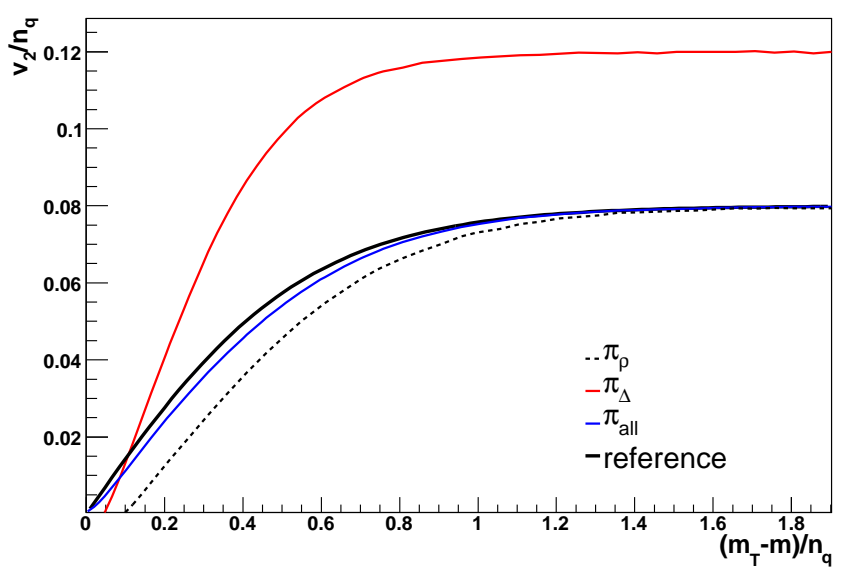

FIG. 5: (color online) Simulation results for pion $v_{2}$ from different sources are shown.

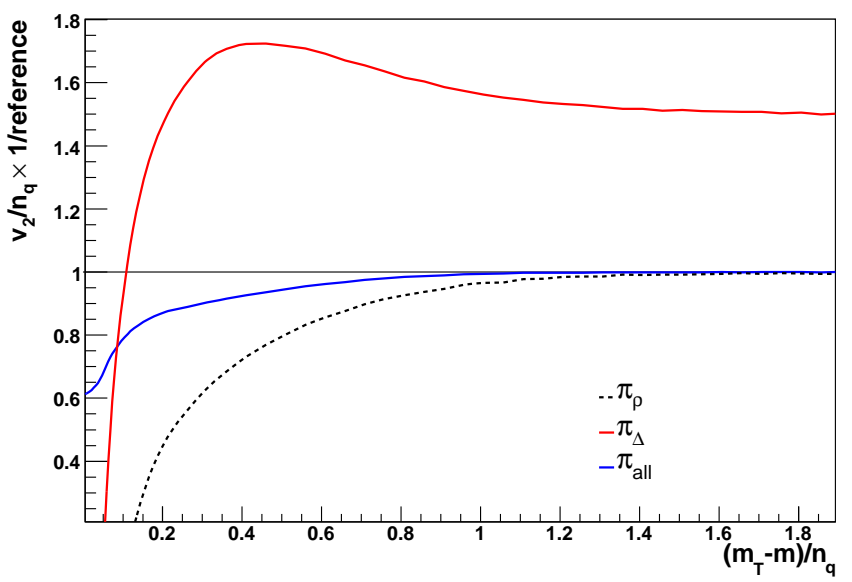

FIG. 6: (color online) The results from Figure 5 are divided by the reference line.

One might naively conclude that the medium is a perfect fluid and is composed of flowing quasi-particles with the quantum numbers of quarks (though yet unidentified mass and width characteristics). Perhaps these quasiparticles even carry the thermodynamic degrees of freedom in their particle form. However, this picture does not sit well with the hydrodynamic paradigm of early time dynamics at RHIC. In the case of a perfect fluid, there can be no well-defined quasi-particles, as their finite mean free path would induce dissipation and viscous effects.

Alternatively, if the medium is not described by a mathematically perfect fluid (i.e. $\eta / s=0$ ), but instead one with a viscosity to entropy density ratio very near the AdS/CFT bound, $\eta / s \approx 1 / 4 \pi$, one might wonder whether or not it is then possible for the medium to contain well defined quasi-particles. Although a rigorous answer to this question is in principle difficult to find, it is possible to derive order of magnitude estimates for the ratio of a quasi-particle's width $\Gamma$, to it's mass, $m$ 
from basic relations. As mentioned previously, a "welldefined" quasi-particle is a mode that can be characterized by a width much smaller than its mass, $\Gamma / m \ll 1$. In the case of a non-relativistic dilute gas [45], one finds that the viscosity $\eta$ is proportional to the product of the average particle momentum, $\langle p\rangle=m \bar{v}$, the number density, $n$, and the particle's mean free path, $\lambda$ :

$$
\eta \sim \frac{1}{3} n \bar{v} m \lambda
$$

In analogy with the thermodynamics of a non-interacting gas of massless bosons, the entropy density is assumed to be proportional to the number density, as $s \sim 4 n$.

To introduce the width of the particle, we identify the mean free path with the particle's lifetime, $\tau$, so that $\lambda=\bar{v} \tau$, and note that $\Gamma$ and $\tau$ are related by $\Gamma=2 / \tau$. Taking the ratio of $\Gamma$ to $m$ with these relationships in place suggests that the quasi particle width is proportional to the temperature, and similar to its mass:

$$
\frac{\Gamma}{m} \approx \frac{16 T}{3 m}
$$

where we have used the Maxwell velocity $\bar{v}=\sqrt{8 T / \pi m}$ to characterize the average quasi-particle speed.

An alternative estimate begins from the expression for the viscosity to entropy density ratio for a weakly coupled quark-gluon plasma derived from kinetic theory in [46],

$$
\left(\frac{\eta}{s}\right)_{w Q G P}=\frac{\lambda T}{5}
$$

Using again the relationships between $\lambda, \tau$, and $\Gamma$, it happens that the width to mass ratio is once more on the order of the temperature, this time

$$
\frac{\Gamma}{m} \approx \frac{8 \pi T}{15 m}
$$

where we have assumed in this case that $\bar{v} \sim c / 3=1 / 3$. In both of these estimates, if we assume that the thermal mass of quasi-particles is $1-3 \cdot T$, then the ratio $\Gamma / m$ is of order one. Thus, if the produced medium has a viscosity near the conjectured bound, it is unlikely that quasi-particle modes would be well defined.

Recently, it has been speculated that the quark-gluon plasma might have a small viscosity and maintain well defined quasi-particles, if there is a dominant contribution from turbulent color fields to the transport coefficients, referred to as an "anomalous viscosity" [47, 48]. This scenario allows for weakly coupled quasi-particles (and thus well-defined) and a large collisional viscosity $\eta_{C}$, but where the total viscosity is determined by the anomalous term, i.e. $\eta^{-1}=\eta_{A}^{-1}+\eta_{C}^{-1}$. We note however that the QED plasma analog is typically non-relativistic, and in the QCD case of the quark-gluon plasma the system is relativistic. One could imagine that the heavy flavor elements of such a QGP plasma would be non-relativistic and thus the compatibility with the QED plasma calculations would be more obvious.

In a relativistic quantum field theory, the sharp distinction between fields and particles does not exist and the factorization of viscosity terms above is non-trivial to mathematically define. Thus, while it is very interesting to experimentally determine the possible size of this anomalous viscosity term, it is unclear if it reconciles the perfect fluid and quasi-particle pictures. Applying non-relativistic calculational insights to the relativistic quark-gluon plasma, including the previous derivations for $\Gamma / m$, is common. However, it is often unclear how to determine which physics effects are actually relevant for describing the observed data.

While quasi-particle widths are large (e.g. while $T>$ $1.05 T_{c}$, which happens to be close to the $T_{c h}$ assumed in most calculations), then hydrodynamics should be the appropriate description of the collision evolution, as is already assumed for RHIC collisions. However, as the temperature approaches $T_{c}$ from above, the widths may become small enough at a temperature $T_{q p}$, such that below this, a quasi-particle transport (QPT) approach would be applicable, with quasi-particles following classical paths. The transition from fluid to quasi-particles would occur at the $T=T_{q p}$ hyper-surface with the fluid $v_{2}$ at that moment. At the end of this this QPT stage, the quasi-particles could form hadrons via recombination.

This quasi-particle transport scenario preceding hadronic transport and then freeze-out allows the simultaneous use of hydrodynamics (at $T>T_{q p}$ ), thermal hadron formation (for $T_{c h}<T<T_{q p}$ ) and even hadronic re-scattering (for $T_{t h}<T<T_{c h}$, e.g. as implemented in Ref. 24]) while allowing in principle for " $n_{q}$ " scaling. With better knowledge of the quasi-particle masses and quantum numbers it could be possible to implement a multi-stage dynamical model incorporating all of these with realistic transport. Of course, the scaling is not inevitable, but depends quite sensitively on the details of the dynamical evolution. For example, if QPT takes place too early, and freeze-out to hadrons occurs rapidly, then hadronic re-scattering may well destroy the " $n_{q}$ " scaling that could have been present just after hadron formation. In addition, if the QPT stage is too short, the uncertainty principle would in fact preclude narrow width quasi-particles.

\section{HADRONIC TRANSPORT AND " $n_{q}$ " SCALING}

In [49], the string and hadron cascade models RQMD and UrQMD provide another means to understand " $n_{q}$ " scaling without hydrodynamics or degrees of freedom carrying valence quark numbers. They consider a picture far from the inviscid fluid limit, comprised of hadrons with long formation times $(\tau \approx 1 \mathrm{fm} / \mathrm{c})$ and transport dynamics driven by various in vacuo interaction cross 
sections. The supposition is that hadron re-scattering encodes the scaling with valence quark number through different Additive Quark Model (AQM) hadron-hadron cross sections. The use of AQM for hadronic cross sections in these transport models is directly motivated by the concept that each constituent quark carries a fraction of the total hadronic cross section. Thus, the constituent quark number scaling it built into these cross sections. It is then immediately seen that " $n_{q}$ " scaling does not require the relevant degrees of freedom to be carried by separate quark-like objects.

Two significant issues pertain to this picture. First, the overall magnitude of the $v_{2}$ is substantially underpredicted by these calculations. Of course, if the formation time is reduced, the final $v_{2}$ value can be made to approach experimental data. As one approaches the hydrodynamic limit where the mean free path is much shorter than the system size, the relative size of the cross sections no longer contribute significant differences in flow. In the hydrodynamic limit, all cross sections need not be the same, but rather, the corresponding mean free paths must all be less than some limiting value. Additionally, in [49] they excluded the $\phi$ and $\Omega$ particles, which have OZI suppressed hadronic cross sections. In this case, the expected cross section is quite different in the scenario of separate quark-like objects from that where they are bound together. Thus, these particles actually represent an excellent test of their hypothesis. Another additional test would be to show the flow for heavy flavor particles (such as D mesons) [39].

We noted earlier that if one shortens the hadron formation time, one can reproduce the overall magnitude of elliptic flow. If such a calculation were to reproduce the experimental data for all hadrons, it might be tempting to conclude the medium dynamics are dominated by hadronic transport, or at least that an ambiguity exists that precludes ruling out this scenario. However, in-medium broadening of resonances is likely to occur, which is due to short time scale interactions (so called "collision broadening"), see for example [50] and references therein. In the case of multiple interactions of resonances on the time scale of $0.1 \mathrm{fm} / c$, the widths would be comparable to the masses and thus the calculation would not be self-consistent in it's treatment of hadrons in a cascade approach.

\section{LIGHT NUCLEI FLOW}

We have also performed a Monte Carlo coalescence calculation for light nuclei and their resulting elliptic flow. In contrast to quark recombination, the mechanism of nucleosynthesis in heavy ion reactions is well understood and the light nuclei wave-functions are known. We parameterize an exploding fireball freeze-out by a Lorentz boosted distribution of nucleons with Boltzmann momen-

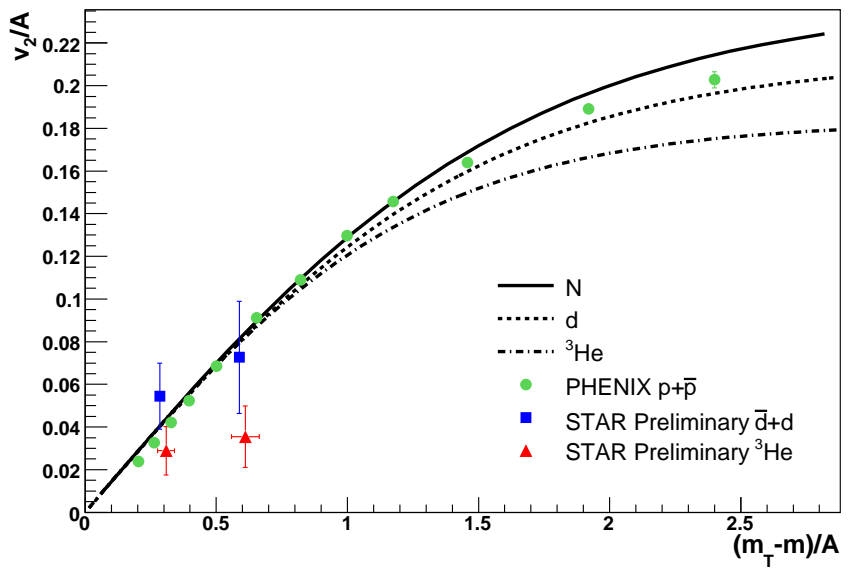

FIG. 7: (color online) Coalescence calculation comparing $v_{2} / n_{A}$ for nucleons (N) and deuterons (d) and ${ }^{3} \mathrm{He}$ for minimum bias $A u+A u$ collisions at $\sqrt{s_{N N}}=200 \mathrm{GeV}$. Preliminary STAR data is shown for $\mathrm{d}$ (squares) and ${ }^{3} \mathrm{He}$ (triangles) and PHENIX data is shown for (anti) protons (circles). [41].

tum and an induced anisotropic flow. We then coalesce these nucleons as in [51], and compute the flow of light nuclei shown in Figure 7. The experimental data for (anti) protons do not follow the reference curve perfectly. This is because the reference line is from Figure 2 and is fit to the charged kaons.

The calculation results for deuterons $\left({ }^{3} \mathrm{He}\right)$ differ from the simple scaling of $v_{2} / A$ versus $\left(m_{T}-m\right) / A$ of order $10 \%(20 \%)$ at high $m_{T}-m$. In fact, this is expected as can be analytically shown in the case of a delta-function in momentum space as the coalescence condition.

$$
v_{2}^{d}\left(p_{T}\right)=\frac{2 v_{2}^{N}\left(p_{T} / 2\right)}{1+2 v_{2}^{N}\left(p_{T} / 2\right)^{2}} \stackrel{v_{2}^{N} \ll 1}{\approx} 2 v_{2}^{N}\left(p_{T} / 2\right)
$$

This is the same as the result from analytic quark coalescence calculations [35, 40]. Given the large $v_{2}$ for protons at large $m_{T}-m$, the correction term in the denominator exactly accounts for the deviation between the calculated curves shown in Figure 7

In the case of light nuclei coalescence where the wavefunction and mechanism are relatively well understood, $A$ (or equivalently $n_{q}$ ) scaling appears to be reasonably well obeyed simply by recombining nucleons close in spacetime and momentum. In this example it would be incorrect to say that the observed scaling (for example of light nuclei $v_{2} / n_{q}$ with $\left(m_{T}-m\right) / n_{q}$ or $v_{2} / A$ with $\left(m_{T}-m\right) / A$ ) stems from baryons being the objects flowing with the medium when the flow is built up. This is not a logical conclusion from the scaling. Rather, the baryons happen to be flowing with the medium at a particular time, and the coalescence mechanism imprints that pattern on the light nuclei.

The situation is quite parallel to quark coalescence in that one cannot conclude that constituent quark degrees 
of freedom were relevant as the flow developed. One only needs these quasi-particles to have the relevant valence quark degrees of freedom and to have the right azimuthal anisotropy as the fluid breaks up. An interesting question is whether one can at minimum conclude that light nuclei (in the light nuclei coalescence case) were not the degrees of freedom at earlier stages. This is not obvious. Just because the coalescence mechanism is consistent with the observed phenomena does not prove that coalescence is the source of light nuclei. For this conclusion to be valid one would have to show that all the features present in light nuclei production are consistent with the coalescence mechanism and that other models for nucleosynthesis fail to describe the empirical results. Similarly to the quark coalescence case, can we exclude bound states (whether hadrons or something more exotic) as the relevant degrees of freedom at earlier stages? The previously discussed UrQMD calculations and uncertainties in the hadronization mechanism leave this as an open question.

Light nuclei were formed in the early universe by processes such as $p+n \longrightarrow d+\gamma$, where the $\gamma$ is required to conserve energy and momentum. In heavy ion reactions these processes have cross sections too small to account for the yield of light nuclei [51]. In this case, the coalescence prescription assumes only that the $p$ and $n$ must overlap with the deuteron wavefunction, and the remaining exchange of energy and/or momentum with the surrounding medium has little impact. In fact, deviations from a common penalty factor for each additional coalesced nucleon has been found to scale with the binding energy per nucleon in the nucleus [52]. This may simply reflect the larger capture cross section for more tightly bound light nuclei, or may be indicative of the required energy and/or momentum exchange with the medium. In the case of quark coalescence, the possible required exchange of energy and/or momentum with the medium is much larger. If the quark-like quasi-particles $\tilde{q}$ have for example mass $1.5 T \approx 255 \mathrm{MeV}$, then the formation process $\tilde{q}+\overline{\tilde{q}} \longrightarrow \pi$ requires a large transfer of energy and/or momentum to the medium. In fact, for all hadrons the exchange is much larger than in the case of light nuclei. At the transition temperature, the mechanism of this exchange as the chiral condensate is re-appearing is not known. As pointed out in [42], the scaling of $v_{2} / n$ with $m_{T}-m$ as opposed to $p_{T}$ may be a consequence of conserving energy instead of momentum in this process, though again there is no a priori reason for this to be the case.

\section{SUMMARY}

In summary, it has been shown that if the matter produced in heavy ion collisions is a near-perfect fluid during the stage when the elliptic flow is built up $(\tau<5-7$ $\mathrm{fm} / \mathrm{c}$ ), then the active thermodynamic degrees of freedom during this time period cannot be associated with specific quasi-particle excitations of the medium. Thus, the determination of $\eta / s$ of the medium during this early time stage is critical to determine whether the medium cannot be described as composed of quasi-particles. If there are no quasi-particles, then the original concept of the quark-gluon plasma as being composed of quarks and gluons with well-defined thermal masses is not realized.

By comparing the empirical observation of " $n_{q}$ " scaling of elliptic flow with hydrodynamic fluid calculations followed by Cooper-Frye hadronization we come to the novel conclusion that at all $\left(m_{T}-m\right)$ " $n_{q}$ " scaling gives better agreement to the experimental data. It has been pointed out that in the region where $v_{2}$ is linearly related to $\left(m_{T}-m\right)$, all values of $\alpha$ would preserve $v_{2} / \alpha$ as linear with $\left(m_{T}-m\right) / \alpha$. However, as shown in Figure 4, typical hydrodynamic models with Cooper-Frye freeze-out do not evince this linear relationship.

We also present results for flow scaling for light nuclei and use this comparison to highlight the similarities and differences of nucleon coalescence into fragments and quark coalescence into hadrons. Just as the light nuclei data does not prove that nucleons were the degrees of freedom when the flow developed, the " $n_{q}$ " scaling is not sufficient evidence that constituent quarks were the degrees of freedom when the flow developed.

We find that if one had knowledge of the relevant dynamical masses and quantum numbers of the ensemble of quasi-particles it would be possible to formulate a multistage approach consisting of: hydrodynamic collective flow, followed by quasi-particle transport (QPT), coalescence into hadrons, hadronic re-scattering and finally freeze-out into free streaming hadrons. This proposal of a QPT stage between the fluid phase and hadronization has yet to be theoretically explored. Most importantly, the masses and widths of the quasi-particles will have to be related to the creation and total duration of the QPT phase.

The authors would like to acknowledge our colleagues (B. Müller, D. T. Son, T. Schaefer, and W. A. Zajc) for useful exchanges, discussions, and suggestions. The authors also thank P. Huovinen for providing the hydrodynamic calculation results. We acknowledge support from the United States Department of Energy grant DEFG02-00ER41152 (L.A.L.L, J.L.N, C.R.) and and DEAC02-98CH10886 (P.S.).

[1] PHEnIX, A. Adare et al., Phys. Rev. Lett. 98, 162301 (2007), nucl-ex/0608033.

[2] E. V. Shuryak, Phys. Rept. 61, 71 (1980).

[3] F. Karsch, (2007), hep-ph/0701210.

[4] U. W. Heinz and M. Jacob, (2000), nucl-th/0002042. 
[5] B. Muller and J. L. Nagle, Ann. Rev. Nucl. Part. Sci. 56, 93 (2006), nucl-th/0602029.

[6] PHENIX, S. Afanasiev et al., (2007), nucl-ex/0703024.

[7] STAR, B. I. Abelev et al., (2007), nucl-ex/0703033.

[8] J. P. Blaizot, E. Iancu, and A. Rebhan, Phys. Rev. Lett. 83, 2906 (1999), hep-ph/9906340.

[9] A. Peshier and W. Cassing, Phys. Rev. Lett. 94, 172301 (2005), hep-ph/0502138.

[10] A. Peshier, Phys. Rev. D70, 034016 (2004), hep$\mathrm{ph} / 0403225$.

[11] E. V. Shuryak and I. Zahed, Phys. Rev. D70, 054507 (2004), hep-ph/0403127.

[12] F. Karsch, S. Ejiri, and K. Redlich, Nucl. Phys. A774, 619 (2006), hep-ph/0510126.

[13] V. Koch, A. Majumder, and J. Randrup, Phys. Rev. Lett. 95, 182301 (2005), nucl-th/0505052.

[14] A. Majumder and B. Muller, Phys. Rev. C74, 054901 (2006), nucl-th/0605079.

[15] G. Baym and C. Pethick, Landau Fermi-Liquid Theory: Concepts and Applications (Wiley, 1992).

[16] J. Eisenstein and H. Stormer, Science 248, 1510 (1990).

[17] P. Huovinen and P. V. Ruuskanen, Ann. Rev. Nucl. Part. Sci. 56, 163 (2006), nucl-th/0605008.

[18] D. Teaney, Phys. Rev. C68, 034913 (2003), nuclth/0301099.

[19] P. Kovtun, D. T. Son, and A. O. Starinets, Phys. Rev. Lett. 94, 111601 (2005), hep-th/0405231.

[20] D. Molnar and M. Gyulassy, Phys. Rev. C62, 054907 (2000), nucl-th/0005051.

[21] M. Bleicher and H. Stoecker, Phys. Lett. B526, 309 (2002), hep-ph/0006147.

[22] M. A. Lisa, S. Pratt, R. Soltz, and U. Wiedemann, Ann. Rev. Nucl. Part. Sci. 55, 357 (2005), nucl-ex/0505014.

[23] F. Cooper and G. Frye, Phys. Rev. D10, 186 (1974).

[24] S. A. Bass and A. Dumitru, Phys. Rev. C61, 064909 (2000), nucl-th/0001033.

[25] D. Teaney, J. Lauret, and E. V. Shuryak, (2001), nuclth/0110037.

[26] STAR, J. Adams et al., Phys. Rev. Lett. 92, 052302 (2004), nucl-ex/0306007.

[27] STAR, J. Adams et al., Phys. Rev. Lett. 95, 122301 (2005), nucl-ex/0504022.

[28] P. Huovinen, Nucl. Phys. A761, 296 (2005), nuclth/0505036.

[29] PHENIX, K. Adcox et al., Nucl. Phys. A757, 184 (2005), nucl-ex/0410003.
[30] PHENIX, A. Adare et al., Phys. Rev. Lett. 98, 172301 (2007), nucl-ex/0611018.

[31] P. Romatschke and U. Romatschke, (2007), arXiv:0706.1522 [nucl-th].

[32] E. Frodermann, R. Chatterjee, and U. Heinz, (2007), arXiv:0707.1898 [nucl-th].

[33] R. J. Fries, B. Muller, C. Nonaka, and S. A. Bass, Phys. Rev. C68, 044902 (2003), nucl-th/0306027.

[34] STAR, J. Adams et al., Nucl. Phys. A757, 102 (2005), nucl-ex/0501009.

[35] D. Molnar and S. A. Voloshin, Phys. Rev. Lett. 91, 092301 (2003), nucl-th/0302014.

[36] T. S. Biro and J. Zimanyi, Phys. Lett. B650, 193 (2007), hep-ph/0607079.

[37] C. R. Allton et al., Phys. Rev. D71, 054508 (2005), heplat/0501030.

[38] V. Greco, (2007), arXiv:0710.0486 [nucl-th].

[39] R. Bellwied, (2007), arXiv:0709.4454 [nucl-ex].

[40] Z.-w. Lin and D. Molnar, Phys. Rev. C68, 044901 (2003), nucl-th/0304045.

[41] H.-d. Liu, (2007), nucl-ex/0701057.

[42] J. Jia and C. Zhang, Phys. Rev. C75, 031901 (2007), hep-ph/0608187.

[43] D. Molnar, (2004), nucl-th/0408044.

[44] V. Greco and C. M. Ko, Phys. Rev. C70, 024901 (2004), nucl-th/0402020.

[45] F. Reif, Fundamentals of Statistical and Thermal Physics (McGraw-Hill Science, 1965).

[46] T. Hirano and M. Gyulassy, Nucl. Phys. A769, 71 (2006), nucl-th/0506049.

[47] M. Asakawa, S. A. Bass, and B. Muller, Prog. Theor. Phys. 116, 725 (2007), hep-ph/0608270.

[48] M. Asakawa, S. A. Bass, and B. Muller, Phys. Rev. Lett. 96, 252301 (2006), hep-ph/0603092.

[49] Y. Lu et al., J. Phys. G32, 1121 (2006), nucl-th/0602009.

[50] J. Lehr and U. Mosel, Phys. Rev. C64, 042202 (2001), nucl-th/0105054.

[51] J. L. Nagle, B. S. Kumar, D. Kusnezov, H. Sorge, and R. Mattiello, Phys. Rev. C53, 367 (1996).

[52] E864, T. A. Armstrong et al., Phys. Rev. Lett. 83, 5431 (1999), nucl-ex/9907002.

[53] Effectively 37 when one calculates the energy density, due to the factor of $7 / 8$ that arises from Fermi-Dirac statistics. 